\title{
Prevalence of Anemia and Compliance With NCCN Guidelines for Evaluation and Treatment of Anemia in Patients With Gynecologic Cancer
}

\author{
Demetra Hypatia Hufnagel, BS1; Sumit Tushar Mehta, $\mathrm{MD}^{2}$; Chinyere Ezekwe, BS; \\ Alaina J. Brown, MD, MPH${ }^{4}$; Alicia Beeghly-Fadiel, PhD, $\mathrm{MPH}^{5}$; and Lauren Shore Prescott, MD, $\mathrm{MPH}^{4}$
}

\begin{abstract}
Background: NCCN recommends evaluation and treatment of all patients with cancer who have anemia. Few studies have evaluated the prevalence of anemia among patients with gynecologic cancer and compliance with the NCCN Clinical Practice Guidelines in Oncology (NCCN Guidelines) for Hematopoietic Growth Factors. Methods: We performed a single-institution retrospective cohort study of patients diagnosed with primary gynecologic cancer between 2008 and 2018. We identified tumor registry-confirmed patients using ICD-O codes from the Synthetic Derivative database, a deidentified copy of Vanderbilt's electronic medical records. Patients were included if they were between ages 18 and 89 years, received initial care at Vanderbilt University Medical Center, and had a hemoglobin measurement within the first 6 months of diagnosis. Anemia was defined as a hemoglobin level $\leq 11 \mathrm{~g} / \mathrm{dL}$ and was graded using CTCAE version 5.0. Results: A total of 939 patients met inclusion criteria, with a median age of 60 years. The most common malignancy was uterine cancer. At the time of cancer diagnosis, 186 patients $(20 \%)$ were noted to have anemia. Within 6 months of diagnosis, 625 patients (67\%) had anemia, of whom 200 (32\%) had grade 3 anemia and 209 (33\%) underwent any evaluation of anemia, including 80 (38\%) with iron studies performed. Of the patients with iron studies performed, 7 (9\%) had absolute iron deficiency and 7 (9\%) had possible functional iron deficiency. Among those with anemia within 6 months of diagnosis, 260 (42\%) received treatment for anemia, including blood transfusion $(n=205 ; 79 \%)$, oral iron $(n=57$; $22 \%)$, intravenous iron $(n=8 ; 3 \%)$, vitamin $B_{12}(n=37 ; 14 \%)$, and folate supplementation $(n=7 ; 3 \%)$. Patients with ovarian cancer were significantly more likely to have anemia and undergo evaluation and treatment of anemia. Conclusions: Anemia is pervasive among patients with gynecologic cancer, but compliance with the NCCN Guidelines is low. Our data suggest that there are opportunities for improvement in the evaluation and management of anemia.
\end{abstract}

J Natl Compr Canc Netw 2021;19(5):513-520 doi: $10.6004 /$ jnccn.2020.7638

${ }^{1}$ Vanderbilt University School of Medicine; ${ }^{2}$ Department of Obstetrics and Gynecology, Vanderbilt University Medical Center; ${ }^{3}$ Meharry Medical College School of Medicine; and ${ }^{4}$ Department of Obstetrics and Gynecology, Division of Gynecologic Oncology, and ${ }^{5}$ Department of Medicine, Division of Epidemiology, Vanderbilt University Medical Center, Nashville, Tennessee.

\section{Background}

Anemia is pervasive among patients with cancer, affecting approximately $30 \%$ to $90 \%$ of those with solid tumors and $26 \%$ to $85 \%$ of those with gynecologic malignancies. ${ }^{1-3}$ The etiology of anemia in patients with cancer is multifactorial, involving tumor-specific and treatment-related elements. ${ }^{1,4,5}$ Chronic inflammation associated with cancer and cancer-related cytokines can mediate the suppression of erythropoietin production and the sequestration of iron, contributing to anemia. Acute blood loss from cytoreductive surgery, renal impairment from chemotherapy, and bone marrow impairment from chemotherapy, radiation, or other treatments may also contribute to cancer-associated anemia. $^{1}$

Anemia in patients with cancer has been associated with increased morbidity and mortality. ${ }^{2,3,6}$ Often presenting as shortness of breath, lethargy, palpitations, or syncope, the symptoms of anemia may be disruptive to daily function and contribute to diminished quality of life. Numerous studies have shown associations between anemia and worse response to treatment and survival in a variety of cancers, including ovarian, endometrial, cervical, and vulvar malignancies. ${ }^{1,7-11}$ Among gynecologic cancers, anemia has been associated with poor local tumor control, potentially related to tumor hypoxiamediated resistance to treatment. ${ }^{1,12}$

Although anemia is prevalent in patients with cancer, evidence suggests that the workup and treatment of anemia in the oncology context is lacking. In a prospective study of 15,000 patients with cancer, $39 \%$ had anemia at initial presentation; however, only $40 \%$ of those with anemia received treatment. ${ }^{2}$ The current NCCN Clinical Practice Guidelines in Oncology (NCCN Guidelines) for Hematopoietic Growth Factors recommend that

\section{See JNCCN.org for supplemental online content.}


patients with a hemoglobin (Hgb) level $\leq 11$ g/dL should undergo prompt evaluation for possible causes of anemia, including iron, nutritional, and hemolysis studies, to guide clinical management. ${ }^{4}$ Our objectives were to determine the prevalence of anemia among patients with gynecologic cancer at Vanderbilt University Medical Center (VUMC) and to assess compliance with the NCCN Guidelines for the evaluation and management of anemia.

\section{Methods}

\section{Study Design and Data Abstraction}

We conducted a retrospective cohort study of all patients with newly diagnosed gynecologic cancers treated at VUMC between 2008 and 2018 using the Synthetic Derivative, a deidentified database of Vanderbilt's electronic medical records created for research purposes. ${ }^{13}$ Tumor registry (TR)-confirmed patients with gynecologic cancer were identified using ICD-O codes. In addition to a TR-confirmed diagnosis of ovarian, fallopian tube, primary peritoneal, uterine, cervical, vulvar, or vaginal cancer, inclusion criteria included age of 18 through 89 years, initial care at our institution, and Hgb measured within \pm 180 days of diagnosis. Exclusion criteria included recurrent disease, dual primary cancer, history of other malignancy, initial care at another institution, noninvasive disease, and borderline tumors of the ovary. Primary tumor site, date of diagnosis, and stage were obtained directly from the TR data. All other data were obtained through either manual review or data extraction within the Synthetic Derivative, with all cancer treatment data restricted to 180 days after TR diagnosis. Initial Hgb level was defined as the Hgb measurement closest to the TR date of diagnosis within 180 days before or after diagnosis, favoring any values before initiation of oncologic treatment. We identified patients with anemia or bleeding diatheses before cancer diagnosis using phenome-wide association study codes and confirmed them through manual chart review. ${ }^{14}$ Prediagnosis oral iron intake was obtained by review of medication records. All other laboratory values and treatment information were abstracted from the 180 days after diagnosis.

\section{Clinical Definitions}

Anemia was defined according to the NCCN Guidelines as $\mathrm{Hgb}$ level $\leq 11 \mathrm{~g} / \mathrm{dL}$. It was graded according to CTCAE version 5.0 as grade 1 ( $\mathrm{Hgb} \leq$ lower limit of normal to $10 \mathrm{~g} / \mathrm{dL}$ ), grade $2(\mathrm{Hgb}<10$ to $8 \mathrm{~g} / \mathrm{dL}$ ), and grade $3(\mathrm{Hgb}$ $<8 \mathrm{~g} / \mathrm{dL}) .{ }^{15}$ Microcytic anemia was defined as mean corpuscular volume $<80 \mathrm{fL}$ and macrocytic anemia was defined as mean corpuscular volume $>100 \mathrm{fL}$. Iron deficiency was defined according to the NCCN Guidelines as follows. Absolute iron deficiency, or the depletion of total body iron stores, corresponded to ferritin level $<30 \mathrm{ng} / \mathrm{mL}$ and transferrin saturation (TSAT) $<20 \%$. Possible functional iron deficiency, or sufficient stored iron with possibly low bioavailable iron, corresponded to ferritin level $>500$ to $800 \mathrm{ng} / \mathrm{mL}$ and TSAT $<50 \%{ }^{4}$ Functional iron deficiency was not determined in our cohort because this condition primarily arises in patients who have received erythropoietin-stimulating agents, which are not in common use among patients with gynecologic cancer at our institution. In accordance with our institution's laboratory reference values, vitamin $B_{12}$ deficiency was defined as levels $<213 \mathrm{pg} / \mathrm{mL}$ and folate deficiency as levels $<5.9 \mathrm{ng} / \mathrm{mL}$. Iron evaluation was defined as at least 1 laboratory test completed for iron level, total iron binding capacity, or ferritin level within 180 days after diagnosis. Other nutritional evaluation was defined as laboratory tests being completed for vitamin $\mathrm{B}_{12}$ or folate level within 180 days after diagnosis. Hemolysis evaluation was defined as laboratory tests for haptoglobin or lactate dehydrogenase level being completed within 180 days after diagnosis. Any anemia evaluation was defined as the presence of any iron evaluation or laboratory tests for vitamin $B_{12}$, folate, lactate dehydrogenase, or haptoglobin level within 180 days after diagnosis. Treatment of anemia was defined as blood transfusion, intravenous iron, oral iron, vitamin $\mathrm{B}_{12}$, or folate supplementation within 180 days after diagnosis.

\section{Statistical Analysis}

Measures of frequency and descriptive statistics were conducted in $\mathrm{R}$ version 3.4.4 (R Foundation for Statistical Computing). Univariate and multivariable regression were performed using SAS 9.4 (SAS Institute Inc) to evaluate the associations between clinical characteristics and anemia prevalence, evaluation, and treatment. Adjustments included age, race, surgery, radiation, chemotherapy, and disease stage. Patients with anemia at initial presentation were included within the group of patients with anemia within 6 months of diagnosis, unless otherwise noted. $P<.05$ was interpreted as statistically significant.

\section{Results}

Baseline and Oncologic Treatment Characteristics A total of 939 patients met inclusion criteria (Table 1). Median age at diagnosis was 60 years (interquartile range, 52-68 years). Most patients were White (88\%) and of non-Hispanic ethnicity (97\%). The most common malignancy was uterine cancer $(n=496 ; 53 \%)$, followed by ovarian $(n=258 ; 27 \%)$, cervical $(n=126 ; 13 \%)$, and 


\section{Table 1. Patient and Treatment Characteristics}

\begin{tabular}{|c|c|}
\hline Characteristic & n (\%) \\
\hline All patients, $\mathrm{N}$ & 939 \\
\hline Age, median (IQR), y & $60(52-68)$ \\
\hline \multicolumn{2}{|l|}{ Race } \\
\hline White & $824(88)$ \\
\hline Black & $92(10)$ \\
\hline Asian & $10(1)$ \\
\hline Other/Unknown & $13(1)$ \\
\hline \multicolumn{2}{|l|}{ Ethnicity } \\
\hline Non-Hispanic & $908(97)$ \\
\hline Hispanic & $15(2)$ \\
\hline Unknown & $16(2)$ \\
\hline \multicolumn{2}{|l|}{ Primary cancer site } \\
\hline Uterus & $496(53)$ \\
\hline Ovary $^{a}$ & $258(27)$ \\
\hline Cervix & $126(13)$ \\
\hline Vulva & $47(5)$ \\
\hline Vagina & $12(1)$ \\
\hline \multicolumn{2}{|l|}{ Disease stage } \\
\hline 1 & $465(50)$ \\
\hline ॥ & $70(7)$ \\
\hline III & $238(25)$ \\
\hline IV & $160(17)$ \\
\hline Unstaged & $6(1)$ \\
\hline \multicolumn{2}{|l|}{ Treatment type ${ }^{b}$} \\
\hline No treatment & $122(13)$ \\
\hline Neoadjuvant chemotherapy & $48(5)$ \\
\hline Adjuvant chemotherapy & $242(26)$ \\
\hline Any chemotherapy & $267(28)$ \\
\hline Radiation & $175(19)$ \\
\hline Surgery & $705(75)$ \\
\hline Patients who underwent surgery, $\mathrm{N}$ & 705 \\
\hline Laparotomy & $358(51)$ \\
\hline Laparoscopy & $265(38)$ \\
\hline Robotic & $13(2)$ \\
\hline Vaginal/Vulvar & $68(10)$ \\
\hline Other & $1(<1)$ \\
\hline Estimated blood loss, median (IQR), mL & $250(100-500)$ \\
\hline
\end{tabular}

Columns may not add up to 100 due to rounding or nonexclusivity of characteristics.

Abbreviation: IQR, interquartile range.

alncluding patients with fallopian tube and primary peritoneal cancer.

bTreatments and procedures are not exclusive.

vulvar or vaginal $(n=59 ; 6 \%)$ cancers. Most patients $(n=535 ; 57 \%)$ presented with early-stage disease and $398(42 \%)$ presented with late-stage disease. A small percentage of patients $(n=58 ; 6.2 \%)$ were noted to have anemia or a bleeding diathesis at any time before TR diagnosis, with most anemias being unspecified (supplemental eTable 1, available with this article at JNCCN. org). Fewer than $3 \%$ of patients had oral iron intake, as determined by medication lists, before TR diagnosis, with the last notation of medication occurring on average 4 years before cancer diagnosis. Fewer than $1 \%$ of all patients had prior oral iron present on medication lists within 1 year of diagnosis. Most patients received treatment for their cancer, including surgery $(n=705$; $75 \%)$, adjuvant chemotherapy $(\mathrm{n}=242 ; 26 \%)$, radiation $(\mathrm{n}=175 ; 19 \%)$, and neoadjuvant chemotherapy $(\mathrm{n}=48$; $5 \%)$. Only $13 \%$ of patients did not undergo any oncologic therapy. Surgical procedure details are listed in supplemental eTable 2 . The median estimated blood loss across all patients who had surgery was $250 \mathrm{~mL}$. This was highest among patients with ovarian cancer $(500 \mathrm{~mL})$ and lowest among those with vulvar and vaginal cancers (100 mL).

\section{Prevalence of Anemia and Anemia Characteristics}

A total of 857 patients had an Hgb measurement taken at initial presentation, with a median $\mathrm{Hgb}$ of $12.7 \mathrm{~g} / \mathrm{dL}$ (Table 2). Of these, 186 (20\%) were noted to have anemia, with a median Hgb of $9.8 \mathrm{~g} / \mathrm{dL}$. Severity of anemia included 87 patients $(47 \%)$ with grade 1 anemia, $76(41 \%)$ with grade 2 anemia, and $23(12 \%)$ with grade 3 anemia. Among the 186 patients who had anemia at initial presentation, $39 \%$ had uterine cancer, $30 \%$ had ovarian cancer, $26 \%$ had cervical cancer, and $5 \%$ had vulvar or vaginal cancer. When the population of patients with anemia at initial presentation was evaluated based on disease site, $15 \%$ of those with uterine cancer, $22 \%$ of those with ovarian cancer, $38 \%$ of those with cervical cancer, and $15 \%$ of those with vulvar or vaginal cancer had anemia at initial presentation. Most patients who had anemia at initial presentation had normocytic anemia $(\mathrm{n}=126 ; 68 \%)$.

Among all patients with cancer, the median nadir Hgb level within 6 months of diagnosis was $9.8 \mathrm{~g} / \mathrm{dL}$. Among patients who had anemia within 6 months of diagnosis $(\mathrm{n}=625 ; 67 \%)$, the median nadir Hgb level was $8.7 \mathrm{~g} / \mathrm{dL}$, with 124 patients $(20 \%)$ with grade 1 anemia, 301 (48\%) with grade 2 anemia, and 200 (32\%) with grade 3 anemia. Similar to the distributions of anemia at initial presentation, most of these patients had normocytic anemia $(n=526 ; 84 \%)$. In addition, among all patients who had anemia within 6 months of diagnosis, $44 \%$ had uterine cancer, $35 \%$ had ovarian cancer, $15 \%$ had cervical cancer, and $6 \%$ had vulvar or vaginal cancer (data not shown). When the population of patients with anemia within 6 months of diagnosis was evaluated based on disease site, $56 \%$ of those with uterine cancer, $86 \%$ of those with ovarian cancer, $72 \%$ of those with cervical 


\section{Table 2. Anemia Characteristics}

\begin{tabular}{|c|c|}
\hline Characteristic & n (\%) \\
\hline All patients, $\mathrm{N}$ & 939 \\
\hline $\mathrm{Hgb}$ at initial presentation, median (IQR), $\mathrm{g} / \mathrm{dL}^{\mathrm{a}}$ & $12.7(11.4-13.7)$ \\
\hline Nadir Hgb, median (IQR), g/dL $b$ & $9.8(8.2-11.7)$ \\
\hline Patients with anemia at initial presentation & $186(20)$ \\
\hline $\mathrm{Hgb}$ at initial presentation, median (IQR), g/dL & $9.8(8.7-10.6)$ \\
\hline \multicolumn{2}{|l|}{ Anemia grade at initial presentation } \\
\hline 1 & $87(47)$ \\
\hline 2 & $76(41)$ \\
\hline 3 & $23(12)$ \\
\hline MCV at initial presentation, median (IQR) & $84(77-89)$ \\
\hline Microcytic & $57(31)$ \\
\hline Normocytic & $126(68)$ \\
\hline Macrocytic & $3(2)$ \\
\hline Patients with anemia within 6 mo of diagnosis & $625(67)$ \\
\hline Hgb at nadir, median (IQR), g/dL & $8.7(7.6-9.8)$ \\
\hline \multicolumn{2}{|l|}{ Anemia grade at nadir } \\
\hline 1 & $124(20)$ \\
\hline 2 & $301(48)$ \\
\hline 3 & $200(32)$ \\
\hline MCV at nadir, median (IQR) & $86(85-93)$ \\
\hline Microcytic & $63(10)$ \\
\hline Normocytic & $526(84)$ \\
\hline Macrocytic & $36(6)$ \\
\hline $\begin{array}{l}\text { Surgical patients with nonanemic } \mathrm{Hgb} \text { at initial } \\
\text { presentation }\end{array}$ & $538(57)$ \\
\hline No anemic $\mathrm{Hgb}$ within 6 mo of diagnosis & $226(42)$ \\
\hline Anemic $\mathrm{Hgb}$ only during postoperative period ${ }^{d}$ & $149(28)$ \\
\hline $\begin{array}{l}\text { Anemic } \mathrm{Hgb} \text { during and after postoperative } \\
\text { periodd }^{\mathrm{d}}\end{array}$ & $120(22)$ \\
\hline Anemic $\mathrm{Hgb}$ only after postoperative period ${ }^{d}$ & $43(8)$ \\
\hline
\end{tabular}

Columns may not add up to 100 due to rounding or nonexclusivity of characteristics.

Abbreviations: Hgb, hemoglobin; IQR, interquartile range; MCV, mean corpuscular volume.

${ }^{a}$ Among patients with pretreatment $\mathrm{Hgb}$ values available $(\mathrm{n}=857)$.

b Lowest Hgb value within 6 mo of diagnosis.

cAmong patients who underwent surgery and had pretreatment $\mathrm{Hgb}$ values available $(n=538)$.

dPostoperative period corresponds to postoperative days 0-30.

cancer, and $59 \%$ of those with vulvar or vaginal cancer had anemia within 6 months of diagnosis (data not shown).

Among 671 patients with pretreatment Hgb values available who did not have anemia at initial presentation, $384(57 \%)$ ultimately developed anemia within 6 months of diagnosis. Among surgical patients, postoperative anemia was common, with 269 (50\%) of surgical patients who initially did not have anemia noted to develop anemia within 30 days of surgery. This persisted beyond the postoperative period, with 120 of these patients remaining anemic or developing a subsequent anemia in the 6 months after diagnosis.

\section{Compliance With NCCN Guidelines}

Among all 939 patients, 241 (26\%) underwent anemia evaluation within the 6 months after diagnosis (Table 3 ). Of these 241 patients, 87 (36\%) underwent evaluation for iron deficiency or anemia of chronic disease with iron studies, 58 (24\%) underwent evaluation for other nutritional causes of anemia, and 167 (69\%) underwent evaluation for hemolysis. Among those who had iron studies completed, 8 (9\%) had absolute iron deficiency and 8 (9\%) had possible functional iron deficiency. Among patients with other nutritional studies completed, $3(5 \%)$ had vitamin $\mathrm{B}_{12}$ deficiency and $6(10 \%)$ had folate deficiency. Among all patients, 283 (30\%) had some treatment for anemia, including blood transfusion $(\mathrm{n}=213 ; 75 \%)$ or iron $(\mathrm{n}=68 ; 24 \%)$, vitamin $\mathrm{B}_{12}(\mathrm{n}=47$; $17 \%)$, or folate supplementation $(n=7 ; 2 \%)$.

Among 625 patients with anemia within the 6 months after diagnosis, 209 (33\%) had any evaluation for anemia, including iron $(\mathrm{n}=80 ; 38 \%)$, other nutritional $(\mathrm{n}=50 ; 24 \%)$, and hemolysis $(\mathrm{n}=146 ; 70 \%)$ studies. Prevalence of absolute and possible functional iron deficiency was low (both 9\%) among patients with anemia who had iron studies performed. Among patients with anemia with other nutritional studies completed, 3 (6\%) had vitamin $\mathrm{B}_{12}$ deficiency and $6(12 \%)$ had folate deficiency. Treatment of anemia was initiated in $260(42 \%)$ patients with anemia. Among these patients, almost half $(\mathrm{n}=124 ; 48 \%)$ had a prior evaluation of anemia. Blood transfusion was the most common intervention $(n=205$; $79 \%)$ followed by iron $(n=62 ; 24 \%)$, vitamin $B_{12}(n=37$; $14 \%)$, and folate supplementation $(n=7 ; 3 \%)$. Approximately $68 \%$ of patients with grade 3 anemia in the 6 months after diagnosis underwent blood transfusion, and $52 \%$ of all transfusions occurred within 7 days before or after surgery. Of 16 patients with possible functional or absolute iron deficiency, 4 (25\%) received iron treatment and 9 (56\%) received any treatment for anemia, including transfusion, iron, or other nutritional supplementation.

\section{Associations With Cancer Site}

Anemia, any evaluation of anemia, iron evaluation, and treatment of anemia were significantly associated with type of gynecologic cancer (Table 4). In multivariable models that included adjustment for age at diagnosis, race, surgery, radiation, chemotherapy, and stage of disease, patients with uterine cancer had approximately $50 \%$ lower odds of having anemia within 6 months of diagnosis, any evaluation of anemia, iron evaluation, any treatment of anemia, or blood transfusion compared with patients with other gynecologic cancers. Patients 


\begin{tabular}{|c|c|c|}
\hline & $\begin{array}{c}\text { All Patients } \\
\text { n (\%) }\end{array}$ & $\begin{array}{c}\text { Patients With Anemia } \\
\text { Within } 6 \text { mo of } \\
\text { Diagnosis } \\
n(\%)\end{array}$ \\
\hline Any anemia evaluation ${ }^{c}$ & $241(26)$ & $209(33)$ \\
\hline \multicolumn{3}{|l|}{ Cancer site $^{d}$} \\
\hline Uterine & $80(33)$ & 65 (31) \\
\hline Ovarian & $122(51)$ & $113(54)$ \\
\hline Cervical & $29(12)$ & $25(12)$ \\
\hline Vulvar & $4(2)$ & $2(1)$ \\
\hline Vaginal & $5(2)$ & $4(2)$ \\
\hline Evaluation for iron deficiency ${ }^{d}$ & $87(36)$ & $80(38)$ \\
\hline Iron deficiency, absolute ${ }^{\mathrm{e}}$ & $8(9)$ & $7(9)$ \\
\hline Iron deficiency, possible ${ }^{e}$ & $8(9)$ & $7(9)$ \\
\hline Other nutritional evaluation ${ }^{d}$ & $58(24)$ & $50(24)$ \\
\hline Vitamin $B_{12}$ deficiency ${ }^{f}$ & $3(5)$ & $3(6)$ \\
\hline Folate deficiency ${ }^{f}$ & $6(10)$ & $6(12)$ \\
\hline Hemolysis evaluation $^{d}$ & $167(69)$ & $146(70)$ \\
\hline Treatment of anemiag & $283(30)$ & $260(42)$ \\
\hline Blood transfusion & $213(75)$ & $205(79)$ \\
\hline Iron supplementation & $68(24)$ & $62(24)$ \\
\hline Oral & $64(23)$ & $57(22)$ \\
\hline Intravenous & $9(3)$ & 8 (3) \\
\hline Vitamin $B_{12}$ supplementation & $47(17)$ & $37(14)$ \\
\hline Oral & $12(4)$ & $8(3)$ \\
\hline Intravenous & $35(12)$ & $29(11)$ \\
\hline Folate supplementation & $7(2)$ & $7(3)$ \\
\hline
\end{tabular}

Columns may not add up to 100 due to rounding or nonexclusivity of characteristics.

${ }^{a}$ Among all patients $(\mathrm{N}=939)$, unless otherwise specified.

${ }^{b}$ Among patients with anemia within 6 mo of diagnosis $(n=625)$, unless otherwise specified.

Including iron studies, nutritional studies (vitamin $B_{12}$, folate), or hemolysis studies.

${ }^{d}$ Among patients with anemia evaluation ( $n=241$ among all patients; $n=209$ among patients with anemia within $6 \mathrm{mo}$ ).

eAmong patients with iron studies available ( $n=87$ among all patients; $n=80$ among patients with anemia within $6 \mathrm{mo}$ ).

${ }^{f}$ Among patients with vitamin $B_{12}$ or folate laboratory values available $(n=58$ among all patients; $\mathrm{n}=50$ among patients with anemia within $6 \mathrm{mo}$ ).

gAmong patients who received treatment for anemia ( $n=283$ among all patients; $\mathrm{n}=260$ among patients with anemia within $6 \mathrm{mo}$ ).

with ovarian cancer had $50 \%$ higher odds of receiving treatment for anemia and more than 2-fold higher odds of having anemia within 6 months of diagnosis, any anemia evaluation, or any iron evaluation compared with patients with other gynecologic cancers. After adjustment of the models, patients with cervical cancer had almost 2-fold higher odds of receiving treatment for anemia and blood transfusion compared with other cancers of interest. Compared with women with other gynecologic cancers, patients with vulvar and vaginal cancer did not have significant associations with odds of anemia or anemia treatment, but did have approximately $60 \%$ lower odds of anemia evaluation.

\section{Associations With Cancer Stage}

A chi-square test of independence was performed to examine the relationship between stage of disease and anemia at initial presentation and within 6 months of diagnosis. Stage of disease and anemia at both time points were significantly associated, with advanced stage (III and IV) being strongly associated with the presence of anemia and early stage being strongly associated with the absence of anemia (initial presentation, $\chi^{2}[3, \mathrm{~N}=851]=69.59 ; P<.001$; within 6 months of diagnosis, $\chi^{2}[3, \mathrm{~N}=933]=117.03 ; P<.001$ ). Maximum grade of anemia within 6 months of diagnosis was similarly associated with cancer stage $\left(\chi^{2}[9, \mathrm{~N}=933]=182.87 ; P<.001\right)$, with early-stage (stage I) disease being strongly associated with no anemia (grade 0) and advanced-stage disease being strongly associated with severe anemia (grade 3).

\section{Discussion}

Anemia in patients with cancer often has a multifactorial etiology, including disease-specific mechanisms such as bone marrow infiltration and inflammation-driven iron sequestration, and treatment-specific mechanisms such as chemotherapy-, immunotherapy-, or radiotherapyinduced myelosuppression or nephrotoxicity. ${ }^{4}$ Prompt recognition, evaluation, and treatment of anemia is critical to optimize outcomes, because anemia is an independent prognostic factor for survival in a variety of cancers. ${ }^{3}$ Given the complex nature of anemia in patients with cancer, the NCCN Guidelines recommend a workup for anemia to include evaluation of the mean corpuscular volume, reticulocyte count, and iron status, and consideration of other causes, such as hemorrhage, hemolysis, nutritional deficiency, renal dysfunction, treatment-induced, inherited, hormonal, or inflammatory. ${ }^{4}$ The NCCN Guidelines also recommend that anemia treatment be guided by the determined cause, although blood transfusion may be considered for asymptomatic patients with comorbidities (eg, cardiac or pulmonary dysfunction) and should be performed for symptomatic patients per AABB guidelines. ${ }^{16}$

In this study, we aimed to determine the prevalence of anemia among patients with gynecologic cancer at our institution and assess compliance with NCCN Guidelines for evaluation of anemia in patients with an Hgb measurement $\leq 11 \mathrm{~g} / \mathrm{dL}$. We found that less than one-third of patients with gynecologic cancers had anemia at initial presentation, but more than half had anemia by 6 months after diagnosis. At both initial presentation and within 6 months of diagnosis, most of the anemia in our 
Table 4. Association of Cancer Site With Anemia, Anemia Evaluation, and Anemia Treatment

\begin{tabular}{|c|c|c|c|c|c|c|c|c|}
\hline & \multicolumn{2}{|c|}{$\begin{array}{l}\text { Uterine vs Other } \\
\text { Gynecologic Cancers }\end{array}$} & \multicolumn{2}{|c|}{$\begin{array}{l}\text { Ovarian vs Other } \\
\text { Gynecologic Cancers }\end{array}$} & \multicolumn{2}{|c|}{$\begin{array}{l}\text { Cervical vs Other } \\
\text { Gynecologic Cancers }\end{array}$} & \multicolumn{2}{|c|}{$\begin{array}{l}\text { Vulvar/Vaginal vs Other } \\
\text { Gynecologic Cancers }\end{array}$} \\
\hline & OR $(95 \% \mathrm{Cl})$ & $P$ Value & OR $(95 \% \mathrm{Cl})$ & $P$ Value & OR $(95 \% \mathrm{Cl})$ & $P$ Value & OR $(95 \% \mathrm{Cl})$ & $P$ Value \\
\hline Unadjusted & $0.48(0.35-0.67)$ & $<.001$ & $1.18(0.82-1.67)$ & .376 & $3.14(2.07-4.74)$ & $<.001$ & $0.80(0.36-1.61)$ & .560 \\
\hline Adjusted $^{a}$ & $0.96(0.65-1.42)$ & .841 & $0.98(0.63-1.54)$ & .836 & $1.53(0.88-2.64)$ & .125 & $0.53(0.23-1.15)$ & .128 \\
\hline Unadjusted & $0.35(0.26-0.46)$ & $<.001$ & $4.06(2.83-6.07)$ & $<.001$ & $1.35(0.90-2.08)$ & .149 & $0.76(0.42-1.24)$ & .225 \\
\hline Adjusteda & $0.58(0.41-0.81)$ & .002 & $2.44(1.58-3.82)$ & $<.001$ & $1.24(0.74-2.13)$ & .417 & $0.66(0.36-1.22)$ & .181 \\
\hline \multicolumn{9}{|c|}{ Any anemia evaluation ${ }^{b}$} \\
\hline Unadjusted & $0.43(0.30-0.61)$ & $<.001$ & $3.36(2.37-4.77)$ & $<.001$ & $0.72(0.43-1.16)$ & .193 & $0.39(0.15-0.90)$ & .042 \\
\hline Adjusted $^{a}$ & $0.52(0.35-0.76)$ & $<.001$ & $3.38(2.15-5.04)$ & $<.001$ & $0.69(0.37-1.27)$ & .241 & $0.39(0.14-0.93)$ & .048 \\
\hline \multicolumn{9}{|c|}{ Any treatment for anemia $a^{b, c}$} \\
\hline Unadjusted & $0.55(0.40-0.76)$ & $<.001$ & $1.81(1.29-2.53)$ & $<.001$ & $1.31(0.83-2.06)$ & .231 & $0.62(0.29-1.25)$ & .195 \\
\hline Adjusted $^{a}$ & $0.62(0.43-0.90)$ & .011 & $1.51(1.01-2.27)$ & .046 & $1.95(1.09-3.48)$ & .023 & $0.57(0.25-1.21)$ & .156 \\
\hline \multicolumn{9}{|c|}{ Blood transfusion ${ }^{b}$} \\
\hline Unadjusted & $0.48(0.34-0.68)$ & $<.001$ & $1.67(1.19-2.36)$ & .003 & $1.58(0.99-2.48)$ & .050 & $0.94(0.43-1.91)$ & .895 \\
\hline Adjusted $^{a}$ & $0.59(0.40-0.88)$ & .010 & $1.24(0.81-1.89)$ & .329 & $2.38(1.30-4.35)$ & .005 & $0.92(0.40-1.96)$ & .828 \\
\hline
\end{tabular}

Bold indicates statistically significant values.

Abbreviation: OR, odds ratio.

${ }^{A}$ Adjusted for age; race; receipt of surgery, radiation, or chemotherapy; and disease stage.

${ }^{b}$ Among patients with anemia within 6 mo of diagnosis $(n=625)$.

Including blood transfusion, iron, vitamin $\mathrm{B}_{12}$, and folate supplementation.

cohort was normocytic. Although normocytic anemia may suggest anemia of chronic disease, bone-marrow suppression, or acute blood loss, we did not see an increase in the prevalence of microcytic anemia by 6 months, as would be expected in chronic blood loss or iron deficiency. Thus, in this cohort, most of the anemia likely occurred because of a combination of chronic disease and treatment-induced myelosuppression. The presence and severity of anemia were significantly associated with cancer stage, with prevalence and grade of anemia increasing with advanced disease stage. Patients with ovarian cancer had significantly higher odds of anemia within 6 months of diagnosis in both the unadjusted and adjusted models. This finding may be accounted for by the higher levels of blood loss during cytoreductive surgery in combination with myelosuppressive adjuvant chemotherapy.

Despite a high prevalence of anemia among all patients with gynecologic cancer, evaluation of anemia was only performed in $33 \%$ of patients with anemia, and treatment for anemia was initiated in $42 \%$ of these patients. Among patients with anemia within 6 months of diagnosis, those with ovarian cancer had higher odds of receiving treatment for anemia. This association was attenuated but remained significant after adjustment for oncologic treatment, possibly because of the frequency with which these patients are monitored as they have follow-up and evaluation during chemotherapy, allowing for more opportunities for intervention. Patients with cervical cancer also had higher odds of receiving treatment for anemia and blood transfusion. This finding may be multifactorial and associated with increased vaginal bleeding at presentation; multimodality treatment that may exacerbate anemia, including surgery, chemotherapy, and radiation; and lower thresholds for transfusion due to the need for radiotherapy, given that anemia is associated with worse outcomes. ${ }^{12}$ Accordingly, higher rates of treatment in this group likely occurred specifically because of higher transfusion rates; among the 48 patients with cervical cancer who initially presented with anemia, 28 (58\%) underwent blood transfusion and only $5(10 \%)$ received iron therapy.

The rates of absolute and possible functional iron deficiency among patients with anemia in our cohort were low ( $18 \%$ total); however, this may be due to the low 
prevalence of patients with available data. A prospective study to determine the true prevalence of iron deficiency in this population would be prudent. Our data indicate that although the prevalence of anemia in patients with gynecologic cancers is high, few patients undergo comprehensive evaluation as recommended by the NCCN Guidelines. Among all patients with gynecologic cancer, this tendency may be influenced by recent clinical trends toward tolerating lower Hgb values to reduce blood transfusion rates or restrictions on the use of erythropoietin-stimulating agents. ${ }^{4,17}$

Our results are in concordance with prior studies of anemia in patients with cancer that have shown frequencies ranging from $30 \%$ to $90 \%$, with both the prevalence and grade of anemia increasing over the 6 months after diagnosis. ${ }^{18-20}$ Although iron deficiency has been reported to occur in up to $40 \%$ of patients with cancer, both inside and outside the context of anemia, we found a lower prevalence in our cohort, although there were low rates of completed iron studies. ${ }^{5,19,21,22}$ In addition, reported rates of iron deficiency vary due to the clinical definitions used; given the links between inflammation and cancer, the ESMO guidelines define iron deficiency as a TSAT $<20 \%$ or ferritin level $<100 \mathrm{ng} / \mathrm{mL}^{23}$ Moreover, some prior studies of iron deficiency in patients with cancer have used a TSAT $<20 \%$ to initially define iron deficiency, with a ferritin level of $<30 \mathrm{ng} / \mathrm{mL}$ corresponding to absolute deficiency and a level of $\geq 30 \mathrm{ng} / \mathrm{mL}$ corresponding to functional deficiency. ${ }^{22}$ When either of these broad definitions of iron deficiency (TSAT $<20 \%$ or ferritin level $<100 \mathrm{ng} / \mathrm{mL}$ ) were applied to patients in our cohort who had iron studies available, these definitions yielded up to $69 \%$ of patients with any iron deficiency. Although perioperative blood transfusion is associated with morbidity and mortality, prior studies have shown relative frequency in gynecologic oncology. ${ }^{17}$ In our cohort, $23 \%$ of all patients underwent blood transfusion, with most transfusions occurring in the perioperative period. In addition to showing opportunities to improve compliance with NCCN Guidelines, our data provide important opportunities to potentially reduce blood transfusion rates by optimizing preoperative anemia evaluation.

The strengths of our study include involvement of a broad, well-characterized cohort of patients with gynecologic cancer over a period of 10 years at a large tertiary-care institution. We determined the prevalence of anemia before treatment where possible and also assessed the prevalence of anemia within 6 months of cancer diagnosis. Few studies have assessed compliance with NCCN Guidelines, and to our knowledge this is the first to assess compliance specifically with NCCN Guidelines for management of anemia among patients with cancer.

This study has several limitations. As a retrospective study utilizing deidentified data, this research may have had incomplete clinical information. The presence of preexisting anemia, bleeding diatheses, and preexisting oral iron intake may have confounded our results, although these numbers are small. Although we only included patients who had Hgb laboratory tests completed at our institution, information regarding further evaluation, treatment, or care from other facilities may have been missing from our record. Information regarding whether hematology consultation was obtained was not available. Furthermore, although we manually reviewed records to confirm that $\mathrm{Hgb}$ levels at initial presentation were recorded prior to surgery or chemotherapy, it was not possible to confirm that these levels were recorded prior to radiation therapy among the $19 \%$ of patients who received this treatment, because specific dates of radiation oncology treatments are managed through a separate system at our institution. The specific impact of radiation on the rates of anemia may be further complicated by the use of radiation to control bleeding from gynecologic malignancies; therefore, radiation may potentially increase or decrease rates of anemia. ${ }^{24}$ Although reticulocyte count may guide anemia evaluation, we were only able to obtain it for $<2 \%$ of our cohort within our study period, and thus did not include it in our analyses.

Previous randomized controlled trials of iron treatment among patients with gynecologic cancer undergoing chemotherapy or chemoradiation have been promising for primary prevention of anemia, treatment of anemia, and reduction of transfusion rates. ${ }^{25-29}$ However, it is unclear whether improvements in quality of life occur with treatment of anemia.. ${ }^{25,30}$ Future work will seek to improve compliance with the NCCN Guidelines for anemia and to assess whether treatment of anemia is associated with improved quality of life and oncologic outcomes. At our institution, a quality improvement project is underway to determine the prevalence of iron deficiency and nutritional anemias and to optimize compliance with the NCCN Guidelines. Potential interventions at our institution include developing laboratory algorithms for reflex iron, vitamin $\mathrm{B}_{12}$, and folate laboratory tests after an anemic Hgb result to facilitate evaluation and guide management, as well as tracking the evaluation of anemia in new patients and those undergoing chemotherapy, who are at increased risk of anemia.

\section{Conclusions}

Anemia is pervasive among patients with gynecologic cancer, and compliance with NCCN Guidelines for the evaluation and treatment of anemia may be low. Our data suggest that there are opportunities for improvement in the evaluation and management of anemia among patients with gynecologic cancer. 
Submitted May 1, 2020; accepted for publication August 11, 2020. Published online February 1, 2021

Author contributions: Study concept and design: Brown, Prescott. Data collection and assembly: Hufnagel, Mehta, Ezekwe. Data analysis and interpretation: Hufnagel, Beeghly-Fadiel, Prescott. Writing - original draft: Hufnagel, Prescott. Table preparation: Hufnagel, Beeghly-Fadiel, Prescott. Writing - review and editing: All authors.

Disclosures: The authors have disclosed that they have not received any financial consideration from any person or organization to support the preparation, analysis, results, or discussion of this article.
Funding: The project described was supported by CTSA award No. UL1TR000445 from the National Center for Advancing Translational Sciences. Its contents are solely the responsibility of the authors and do not necessarily represent official views of the National Center for Advancing Translational Sciences or the National Institutes of Health

Correspondence: Lauren Shore Prescott, MD, MPH, Department of Obstetrics and Gynecology, Division of Gynecologic Oncology, Vanderbilt University Medical Center, B1100 Medical Center North, Nashville, TN 37232-2516. Email: lauren.prescott@vumc.org

\section{References}

1. Knight K, Wade S, Balducci L. Prevalence and outcomes of anemia in cancer: a systematic review of the literature. Am J Med 2004;116(Suppl 7A):11S-26S.

2. Birgegård G, Aapro MS, Bokemeyer C, et al. Cancer-related anemia: pathogenesis, prevalence and treatment. Oncology 2005;68(Suppl 1): 3-11.

3. Caro JJ, Salas M, Ward A, et al. Anemia as an independent prognostic factor for survival in patients with cancer: a systemic, quantitative review. Cancer 2001;91:2214-2221.

4. Becker PS, Griffiths EA, Alwan, L, et al. NCCN Clinical Practice Guidelines in Oncology: Hematopoietic Growth Factors. Version 2.2020. Accessed May 1, 2020. To view the most recent version, visit NCCN.org

5. Gilreath JA, Stenehjem DD, Rodgers GM. Diagnosis and treatment of cancer-related anemia. Am J Hematol 2014;89:203-212.

6. Prescott LS, Aloia TA, Brown AJ, et al. Perioperative blood transfusion in gynecologic oncology surgery: analysis of the National Surgical Quality Improvement Program Database. Gynecol Oncol 2015;136: 65-70.

7. Münstedt K, Kovacic M, Zygmunt M, et al. Impact of hemoglobin levels before and during chemotherapy on survival of patients with ovarian cancer. Int J Oncol 2003;23:837-843.

8. Shin NR, Lee YY, Kim SH, et al. Prognostic value of pretreatment hemoglobin level in patients with early cervical cancer. Obstet Gynecol Sci 2014;57:28-36

9. Hierlihy P, Jenkin RD, Stryker JA. Anemia as a prognostic factor in cancer of the cervix: a preliminary report. Can Med Assoc J 1969;100: 1100-1102

10. Njølstad TS, Engerud H, Werner HMJ, et al. Preoperative anemia, leukocytosis and thrombocytosis identify aggressive endometrial carcinomas. Gynecol Oncol 2013;131:410-415.

11. Tamussino KF, Gücer F, Reich $O$, et al. Pretreatment hemoglobin, platelet count, and prognosis in endometrial carcinoma. Int J Gynecol Cancer $2001 ; 11: 236-240$.

12. Grogan M, Thomas GM, Melamed I, et al. The importance of hemoglobin levels during radiotherapy for carcinoma of the cervix. Cancer 1999;86: 1528-1536.

13. Roden DM, Pulley JM, Basford MA, et al. Development of a large-scale de-identified DNA biobank to enable personalized medicine. Clin Pharmacol Ther 2008;84:362-369.

14. Denny JC, Bastarache L, Ritchie MD, et al. Systematic comparison of phenome-wide association study of electronic medical record data and genome-wide association study data. Nat Biotechnol 2013;31: 1102-1110.

15. National Cancer Institute. Common Terminology Criteria for Adverse Events (version 5.0). Accessed April 30, 2020. Available at: https://ctep.cancer.gov/protocolDevelopment/electronic_applications/ ctc.htm
16. Carson JL, Guyatt G, Heddle NM, et al. Clinical practice guidelines from the AABB: red blood cell transfusion thresholds and storage. JAMA 2016 316:2025-2035.

17. Prescott LS, Taylor JS, Enbaya A, et al. Choosing wisely: decreasing the incidence of perioperative blood transfusions in gynecologic oncology. Gynecol Oncol 2019;153:597-603.

18. Ludwig $\mathrm{H}$, Van Belle $\mathrm{S}$, Barrett-Lee $\mathrm{P}$, et al. The European Cancer Anaemia Survey (ECAS): a large, multinational, prospective survey defining the prevalence, incidence, and treatment of anaemia in cancer patients. Eur J Cancer 2004;40:2293-2306.

19. Busti F, Marchi G, Ugolini S, et al. Anemia and iron deficiency in cancer patients: role of iron replacement therapy. Pharmaceuticals (Basel) 2018 11:94.

20. $\mathrm{Xu} \mathrm{H}, \mathrm{Xu} \mathrm{L}$, Page JH, et al. Incidence of anemia in patients diagnosed with solid tumors receiving chemotherapy, 2010-2013. Clin Epidemiol 2016;8: 61-71.

21. Rodgers GM, Gilreath JA. The role of intravenous iron in the treatment of anemia associated with cancer and chemotherapy. Acta Haematol 2019 , 142:13-20.

22. Ludwig $H$, Müldür $E$, Endler $G$, et al. Prevalence of iron deficiency across different tumors and its association with poor performance status, disease status and anemia. Ann Oncol 2013;24:1886-1892.

23. Aapro M, Beguin Y, Bokemeyer C, et al. Management of anaemia and iron deficiency in patients with cancer: ESMO Clinical Practice Guidelines. Ann Oncol 2018;29(Suppl 4):iv96-110.

24. Sapienza LG, Ning MS, Jhingran A, et al. Short-course palliative radiation therapy leads to excellent bleeding control: a single centre retrospective study. Clin Transl Radiat Oncol 2018;14:40-46.

25. Dangsuwan $P$, Manchana T. Blood transfusion reduction with intravenous iron in gynecologic cancer patients receiving chemotherapy. Gynecol Oncol 2010;116:522-525.

26. Athibovonsuk P, Manchana T, Sirisabya N. Prevention of blood transfusion with intravenous iron in gynecologic cancer patients receiving platinumbased chemotherapy. Gynecol Oncol 2013;131:679-682.

27. Kim YT, Kim SW, Yoon BS, et al. Effect of intravenously administered iron sucrose on the prevention of anemia in the cervical cancer patients treated with concurrent chemoradiotherapy. Gynecol Oncol 2007;105: 199-204.

28. Steinmetz $T$, Totzke $U$, Schweigert $M$, et al. A prospective observational study of anaemia management in cancer patients-results from the German Cancer Anaemia Registry. Eur J Cancer Care (Engl) 2011;20: 493-502.

29. Steinmetz HT. The role of intravenous iron in the treatment of anemia in cancer patients. Ther Adv Hematol 2012;3:177-191.

30. Steinmetz T, Schröder J, Plath $M$, et al. Antianemic treatment of cance patients in German routine practice: data from a prospective cohort study_the Tumor Anemia Registry. Anemia 2016;2016:8057650. 
Supplemental online content for:

Prevalence of Anemia and Compliance With NCCN Guidelines for

\section{Evaluation and Treatment of Anemia in Patients With Gynecologic Cancer}

Demetra Hypatia Hufnagel, BS; Sumit Tushar Mehta, MD; Chinyere Ezekwe, BS; Alaina J. Brown, MD, MPH; Alicia Beeghly-Fadiel, PhD, MPH; and Lauren Shore Prescott, MD, MPH

J Natl Compr Canc Netw 2021;19(5):513-520

eTable 1: Comorbidities or Oral Iron Intake Before Cancer Diagnosis eTable 2: Procedures Among Patients Who Underwent Surgery 


\begin{tabular}{|c|c|}
\hline Characteristic & $n(\%)$ \\
\hline Any PheWAS code ${ }^{a}$ & $58(6.2)$ \\
\hline 280: Iron deficiency anemias & $9(<1.0)$ \\
\hline 281.11: Pernicious anemia & $1(<1.0)$ \\
\hline 281.12: Other vitamin $B_{12}$ deficiency anemia & $1(<1.0)$ \\
\hline 282: Hereditary hemolytic anemias & $2(<1.0)$ \\
\hline 284: Aplastic anemia & $3(<1.0)$ \\
\hline 285: Other anemias & $16(1.7)$ \\
\hline 285.1: Acute posthemorrhagic anemia & $9(<1.0)$ \\
\hline 285.21: Anemia in chronic kidney disease & $4(<1.0)$ \\
\hline 286.7: Other and unspecified coagulation defects & $9(<1.0)$ \\
\hline 286.9: Abnormal coagulation profile & $4(<1.0)$ \\
\hline Oral iron & $24(2.6)$ \\
\hline
\end{tabular}

Columns may not add up to 100 due to rounding or nonexclusivity of characteristics.

Abbreviation: PheWAS, phenome-wide association study.

aPheWAS codes without any results included 280.1: iron deficiency anemias, unspecified or not due to blood loss; 280.2: iron deficiency anemia secondary to blood loss (chronic); 281: other deficiency anemia; 281.1: megaloblastic anemia; 281.13: folate-deficiency anemia; 281.9: deficiency anemias; 282.5: sickle cell anemia; 282.9: other hereditary hemolytic anemias; 283: acquired hemolytic anemias; 283.1: autoimmune hemolytic anemias; 283.2 : nonautoimmune hemolytic anemias; 284.2: constitutional aplastic anemia; 285.2: anemia of chronic disease; 285.3: sideroblastic anemia; 286: coagulation defects; 286.1: congenital coagulation defects; 286.4: acquired coagulation factor deficiency; 286.5: hemorrhagic disorder because of intrinsic circulating anticoagulants.

\section{eTable 2. Procedures Among Patients Who} Underwent Surgery $(n=705)$

\begin{tabular}{|lc|}
\hline Surgical Procedure & $\mathbf{n}(\%)$ \\
\hline Hysterectomy & $513(73)$ \\
\hline Unilateral salpingo-oophorectomy & $36(5)$ \\
\hline Bilateral salpingo-oophorectomy & $271(38)$ \\
\hline Omentectomy & $201(29)$ \\
\hline Pelvic lymph node dissection & $265(38)$ \\
\hline Para-aortic lymph node dissection & $227(32)$ \\
\hline Debulkingb & $131(65)$ \\
\hline Bowel resection & $30(4)$ \\
\hline Appendectomy & $29(4)$ \\
\hline Exenteration & $6(<1)$ \\
\hline Vaginal or vulvar excision & $36(5)$ \\
\hline Other & $4(<1)$ \\
\hline
\end{tabular}

Columns may not add up to 100 due to rounding or nonexclusivity of characteristics. aprocedures are not exclusive.

${ }^{b} A m o n g$ patients with ovarian cancer who underwent surgery $(n=203)$. 